\title{
Original Research \\ Construction of a pyroptosis-related classifier for risk prediction of acute myocardial infarction
}

\author{
Kehang Guo ${ }^{1,2, \dagger}$, Zewei Zhuo ${ }^{2,3, \dagger}$, Pengfei Chen ${ }^{4, \dagger}$, Huihuan $\mathrm{Wu}^{1,2}$, Qi Yang ${ }^{2}$, Jingwei Li ${ }^{2}$, \\ Rui Jiang ${ }^{2,3}$, Qiuxian Mao ${ }^{5, *}$, Hao Chen ${ }^{1,2, *}$, Weihong Sha ${ }^{1,2, *}$ \\ ${ }^{1}$ School of Medicine, South China University of Technology, 510030 Guangzhou, Guangdong, China \\ ${ }^{2}$ Department of Gastroenterology, Guangdong Provincial People's Hospital, Guangdong Academy of Medical Sciences, 510080 Guangzhou, \\ Guangdong, China \\ ${ }^{3}$ School of Bioscience and Bioengineering, South China University of Technology, 510006 Guangzhou, Guangdong, China \\ ${ }^{4}$ Department of Laboratory Medicine, State Key Laboratory of Oncology in South China, Collaborative Innovation Center for Cancer Medicine, Sun \\ Yat-sen University Cancer Center, 510060 Guangzhou, Guangdong, China \\ ${ }^{5}$ Prenatal Diagnostic Department, Guangdong Second Provincial General Hospital, 510317 Guangzhou, Guangdong, China \\ *Correspondence: maoqx@gd2h.org.cn (Qiuxian Mao); chenhao@gdph.org.cn (Hao Chen); shaweihong@gdph.org.cn (Weihong Sha) \\ ${ }^{\dagger}$ These authors contributed equally. \\ Academic Editors: Brian Tomlinson and Takatoshi Kasai \\ Submitted: 20 December 2021 Revised: 13 January 2022 Accepted: 21 January 2022 Published: 9 February 2022
}

\begin{abstract}
Background: Acute myocardial infarction (AMI) is a common cardiovascular disease that has a high mortality. Pyroptosis is a programmed cell death mediated by inflammasome. It remains to be clarified on the expression pattern and risk predictive role of pyroptosisrelated genes in AMI. Methods: The gene expression data were extracted from the Gene Expression Omnibus (GEO), and pyroptosisrelated genes were obtained from published articles. Pyroptosis-related differential expressed genes were selected between normal and AMI samples and then we explored their immune infiltration level using CIBERSORT. Univariate Cox and LASSO regression were applied to establish a classifier based on pyroptosis-related genes. ROC analysis was utilized to evaluate the classifier. Results: In this study, we obtained 20 pyroptosis-related genes which showed differential expression in AMI and normal samples. Among the differential expressed genes, $G Z M B$ was significantly positively associated with activated NK cells $(\mathrm{R}=0.71, p<0.01)$, while NLRP3 exhibited a negative correlation with resting NK cells $(\mathrm{R}=-0.66, p<0.01) .9$ genes (NLRP9, GSDMD, CASP8, AIM2, GPX4, NOD1, NOD2, $S C A F 11, G S D M E)$ were eventually identified as a predictive risk classifier for AMI patients. With the classifier, patients at high and low risk could be discriminated. Further external validation showed the high accuracy of the classifier (AUC $=0.75$ ). Conclusions: Pyroptosis-related genes are closely related to immune infiltration in AMI, and a 9-gene classifier has good performance in predicting the risk of AMI with high accuracy, which could provide a new way for targeted treatment in AMI.
\end{abstract}

Keywords: acute myocardial infarction; pyroptosis; immune infiltration; risk prediction; classifier

\section{Introduction}

Acute myocardial infarction (AMI) is the most prevalent disease with high mortality in the world. Statistically, it could result in a death toll accounting for $20 \%$ of all global deaths. It has an annually increasing incidence predicted to $0.44-1.42 \%$, and causes $4.2-13.5 \%$ in-hospital mortality $[1,2]$. For the past few years, multiple treatments, such as reperfusion, percutaneous coronary intervention (PCI) and anti-thrombotic, have brought survival benefits in this patient cohort and slightly decreased the related mortality $[3,4]$. However, AMI remains a great threat to human health and imparts a large economic burden in the world [5]. Serum biomarkers, such as creatine kinase-MB, cardiac troponin I, and cardiac troponin $\mathrm{T}$, are proven a diagnostic indicator for AMI of clinical significance [6,7]. Nevertheless, they are not only lack of the predictive value, but also not perfectly specific biomarkers for AMI $[8,9]$. Currently, there is still a lack of specific biomarkers capable of identi- fying patients at a high risk of AMI in early stages.

Pyroptosis is a type of pro-inflammatory regulated cell death dependent on gasdermin family of proteins [10]. It causes cell death by cell lysis, a result of impaired cellular osmotic pressure balance because of the formation of cell membrane pores, with the involvement of NLRP3/Caspase $1 / G S D M D$ axis [11]. It is reported that pyroptosis plays a vital role in cardiovascular diseases, notably AMI. NLRP3 inflammasome and Caspase-1 are markedly up-regulating in AMI $[12,13]$. At present, several studies have found that suppressing pyroptosis of cardiomyocytes can dramatically reduce myocardial infarction [14,15]. Several recent studies have revealed the prognostic value of a novel pyroptosisrelated genes signature in various diseases $[16,17]$.

Based on the existing studies, pyroptosis plays an essential role in the process of occurrence and development of the AMI. However, whether pyroptosis is predictive for the risk of AMI is largely unknown and requires further exploration. Hence, we firstly screened genes associ- 
ated with pyroptosis from the AMI gene expression profile obtained from Gene Expression Omnibus (GEO) and then analyzed their association with infiltrating immune cells. A risk prediction classifier for AMI was devised. Finally, we detected the prediction accuracy through an external cohort, which may help better clinical application.

\section{Methods}

\subsection{Data collection}

GSE59867 microarray documented with AMI gene expression data was downloaded from GEO database (http s://www.ncbi.nlm.nih.gov/geo/). The microarray data were from GPL6244 platform (HuGene-1_0-st Affymetrix human genome 1.0 ST), including 390 AMI samples and 46 healthy control samples. GSE62646 microarray was additionally obtained as the validation set. Platform annotation files were downloaded to annotate Gene Symbol with Probe ID. Mean probe expression was regarded as a gene expression if the Gene Symbol could be annotated by multiple Probe IDs. In that way, gene expression profiles of the AMI and normal samples were obtained. Referring to existing literature [18] and the MSIGDB database (http://www.gs ea-msigdb.org/gsea/msigdb/), 41 pyroptosis-related genes were searched and 38 of them were overlapped with the genes from GSE59867.

\subsection{Differential analysis}

Pyroptosis-related genes were analyzed by "limma" package in AMI and normal samples. Genes that met $|\log \mathrm{FC}| \geq 0$ and adjust $p<0.05$ (Benjamini-Hochberg corrected) were screened as differential expressed genes (DEGs). R package "ggpubr" was used to make a Volcano Plot, and package "pheatmap" was operated to obtain a Heat Map [19].

\subsection{Correlation analysis}

The association between pyroptosis-related genes in AMI and all samples was analyzed by Pearson correlation analysis according to a Bubble Chart by R package "corrplot" and Scatter, Density Plots by package "ggplot".

\subsection{Immune microenvironment}

CIBERSORT is used to provide the absolute proportions of 22 infiltrating immune cells in the immune microenvironment based on gene expression data. It is a type of deconvolution algorithm with the support vector regression analysis in a set of reference genes $(n=547)$ [20]. By this algorithm, each sample can be conferred a $p$ value ( $p$ $<0.01)$ to assess the reliability of the deconvolution. Here, CIBERSORT package was obtained from the developer and applied with the default feature matrix. The associations between the 22 infiltrating immune cells were further visualized by R package "corrplot".

\subsection{Filtering of redundant factors by LASSO and construction of a risk prediction model}

Univariate Cox analysis was devised to filter redundant genes from the DEGs. LASSO regression model was established to further screen the remaining genes [21], and the genes of significant predictive value for AMI were taken to analyse in a multi-variate analysis. A riskscore was thus obtained and formulated as: riskscore $=\sum_{i=1}^{n} \beta_{i} *$ $\operatorname{Exp}(i)$ (i= the number of key genes) [22].

With the sextile as the threshold, patients were respectively assigned to high and low risk groups. An external validation set was used to test the model, and receiver operating characteristic (ROC) curve was plotted to assess the predictive performance. To further illuminate the linkage mechanisms between AMI onset and pyroptosis-related genes, a figure was presented.

\subsection{Data analysis}

R v4.0.2(R Foundation for Statistical Computing, Vienna, Austria, http://www.r-project.org/) and RStudio v1.2.1335 (Integrated Development for R. RStudio, Inc., Boston, MA, USA, http:/www.rstudio.com/) were operated to perform data analysis. Significance evaluation was fulfilled by $t$-test or Mann-Whitney test. Spearman correlation coefficient was calculated to identify the associations between the pyroptosis-related genes and 22 infiltrating immune cells. Cox proportional hazard regression model was established to estimate hazards ratio (HR) along with $95 \%$ confidence interval (CI). ROC curve was made to estimate the accuracy of the model in predicting the risk of AMI. All differences on $p<0.05$ were regarded to have statistical significance.

\section{Results}

\subsection{Pyroptosis-related DEGs}

Gene expression profiles from the whole blood samples in 390 AMI cases and 46 healthy controls were obtained from the GSE59867 microarray. Following differential analysis and combining the 41 reported pyroptosisrelated genes, finally 20 pyroptosis-related DEGs were identified, including 13 up-regulated genes (NLRP9, GSDMC, NLRC4, GSDMD, CASP9, ELANE, GPX4, NLRP3, NLRP6, NOD1, NOD2, PYCARD, GSDME) and 7 downregulated genes (DHX9, GZMA, GZMB, GSDMB, CASP8, AIM2, SCAF11) (Fig. 1A). Fig. 1B shows the differential expression of the 20 DEGs in AMI and normal samples. Fig. $1 \mathrm{C}$ presents the heatmap of the 20 DEGs.

\subsection{Correlation analysis between DEGs}

The "corrplot" package was used to perform correlation analysis in the pyroptosis-related DEGs in both all included samples and AMI samples. Results revealed that GZMA and GZMB were significantly associated in both two cohorts (All: 0.71, AMI: 0.69) (Fig. 2). 
A

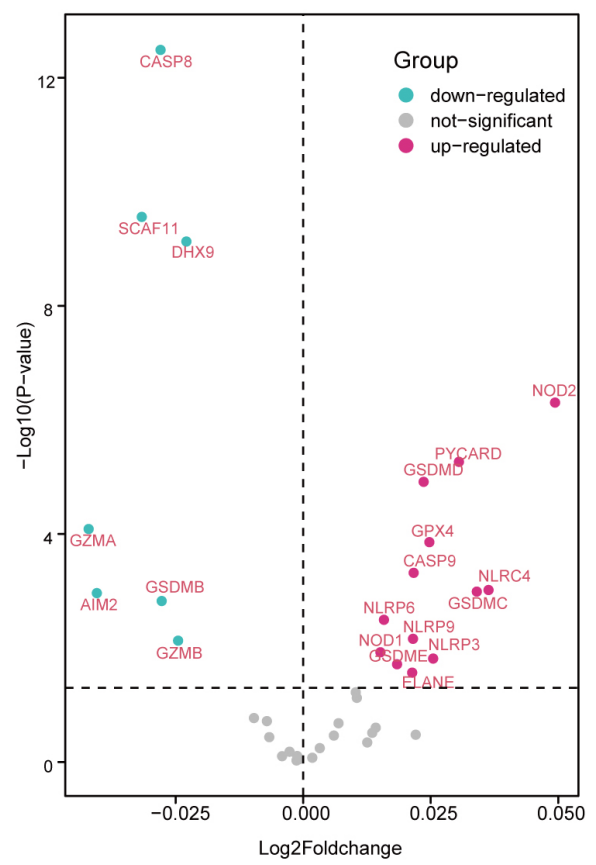

B

group 官 MI 追 NC
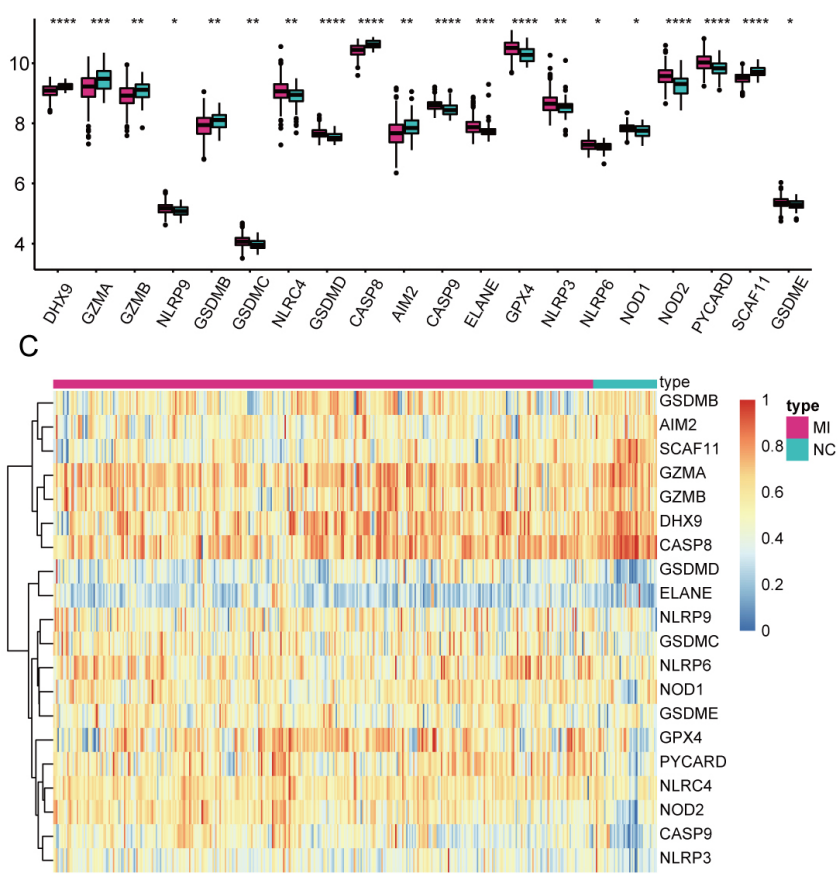

Fig. 1. DEGs associated with pyroptosis in AMI. (A) Volcano Plot for the DEGs. (B) Box Plots showing the differential gene expression in AMI and normal samples. (C) Heatmap for the DEGs. ${ }^{*} p<0.05, * * p<0.01, * * * p<0.001$.

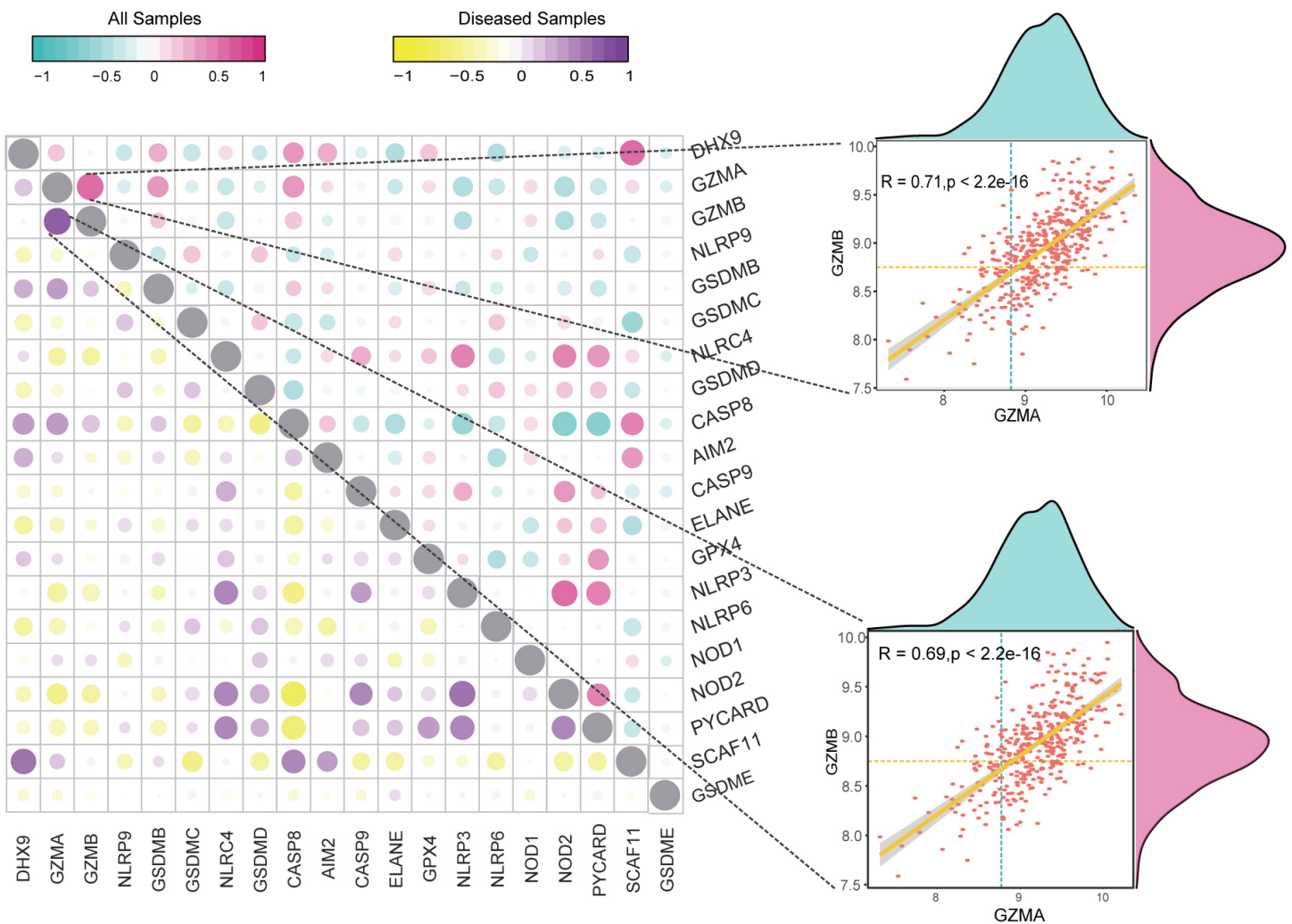

Fig. 2. Correlation analysis on pyroptosis-related DEGs. Scatter Plot and Density Plot showing the correlation between $G Z M A$ and GZMB in all samples and AMI samples. 


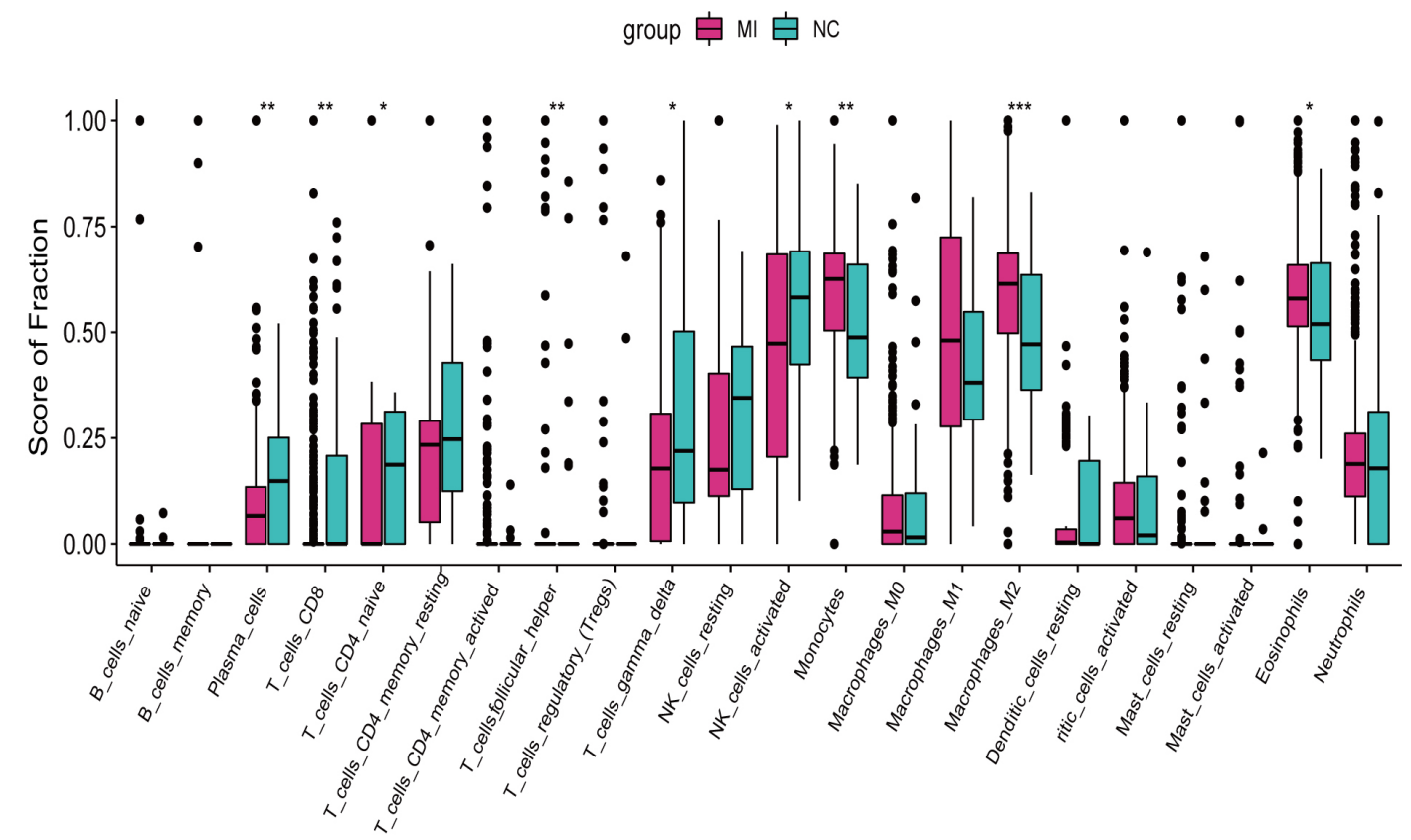

Fig. 3. Infiltrating immune cells in AMI and normal samples. Red for normal samples and Green for AMI samples. $* p<0.05$, ** $p<0.01,{ }^{* * *} p<0.001$.

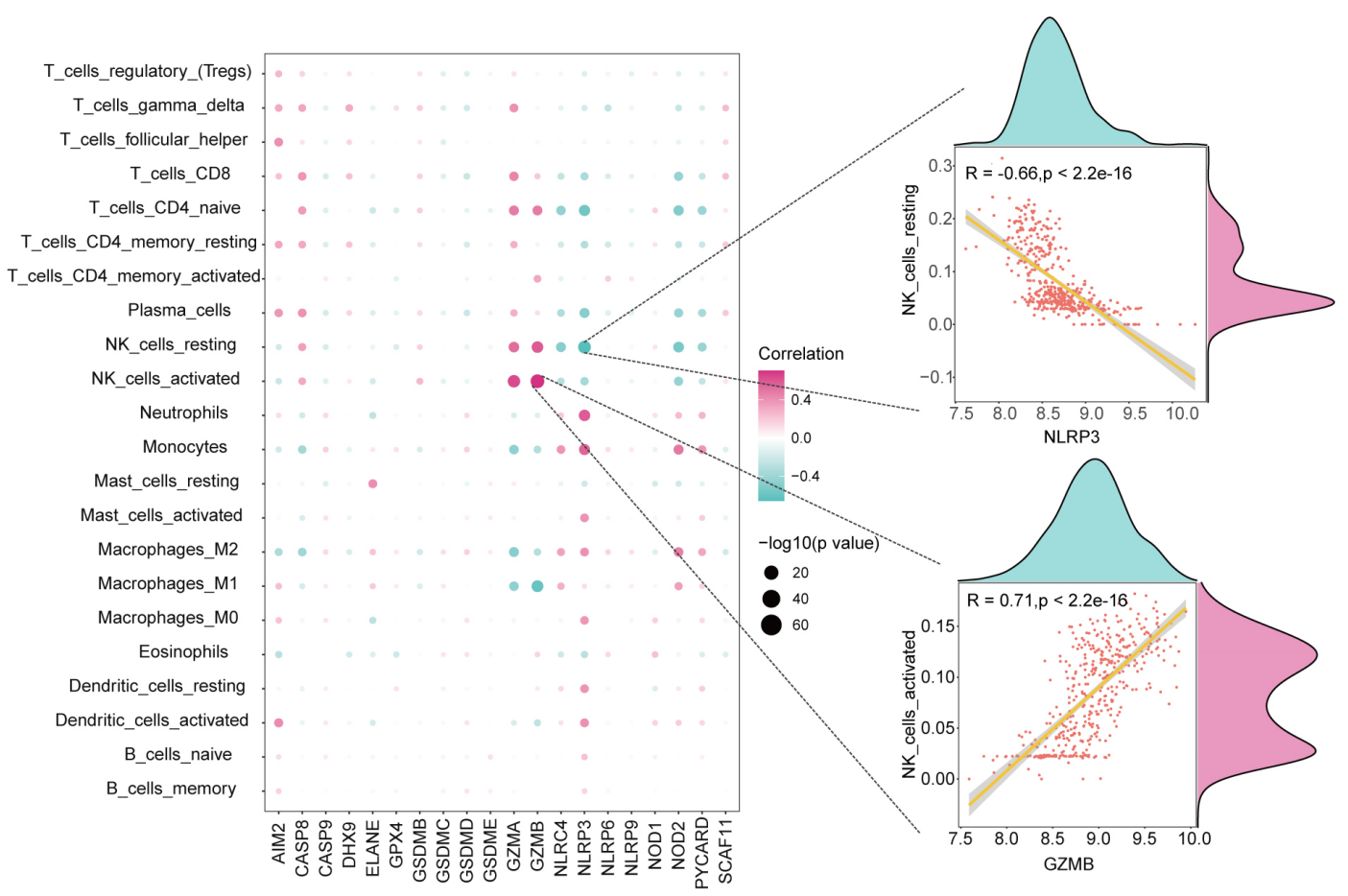

Fig. 4. Correlation analysis between pyroptosis-related DEGs and immune cells. Scatter Plot and Density Plot showing the associations between GZMB and NK_cells_activated, NLRP3 and NK_cells_resting.

\subsection{Association between pyroptosis-related DEGs and infiltrating immune cells}

Plasma cells, T cells CD8, T cells follicular helper were found more abundant in AMI samples versus the healthy control $(p<0.01)$, while Monocytes and Macrophages M2 were reversely higher in normal samples $(p<0.01)$ (Fig. 3). Additionally, remarkable asso- ciations were indicated in infiltrating immune cells and the pyroptosis-related DEGs. Of note, GZMB was evidently correlated to NK cells activated in a positive manner $(\mathrm{R}=$ $0.71, p<2.2 \mathrm{e}-16)$, and NLRP3 was remarkably negatively associated with NK cells resting $(\mathrm{R}=-0.66, p<2.2 \mathrm{e}-16)$ (Fig. 4). 


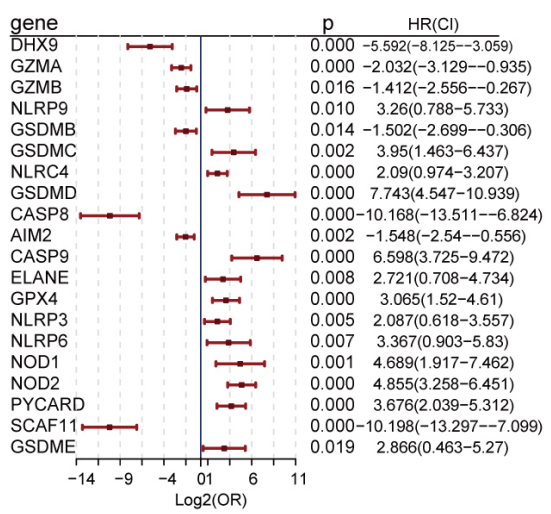

D

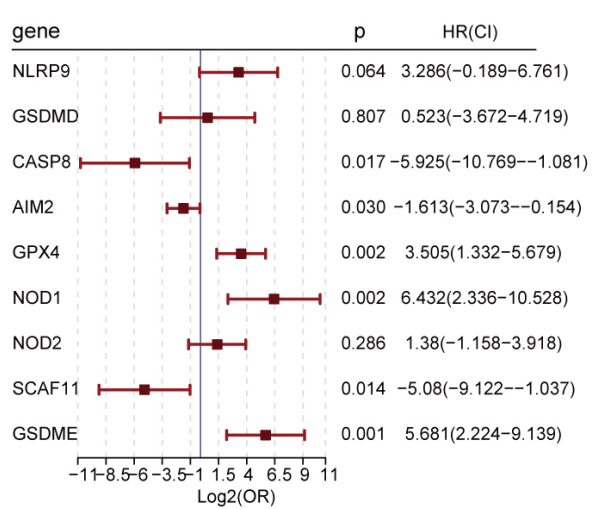

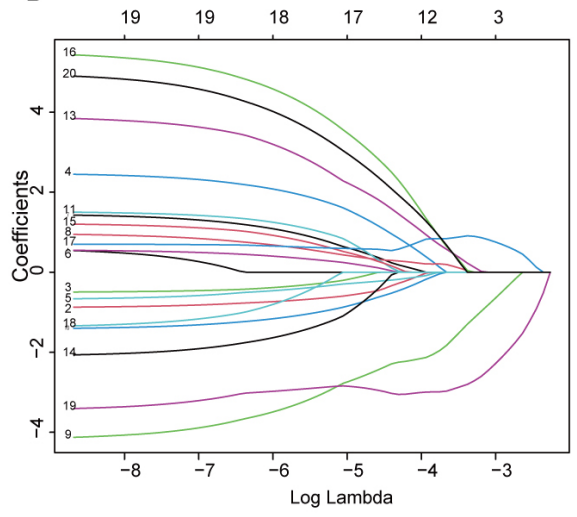

E

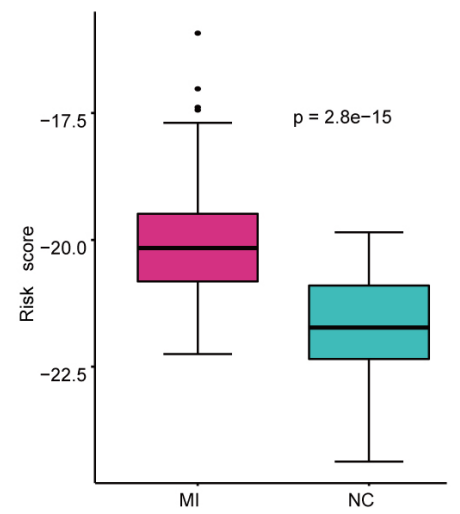

C

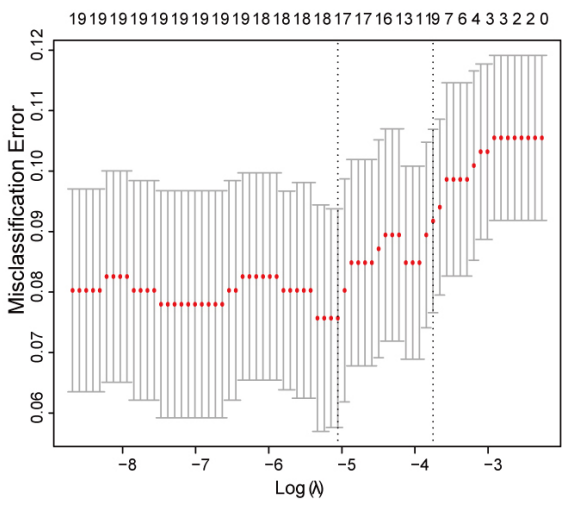

F

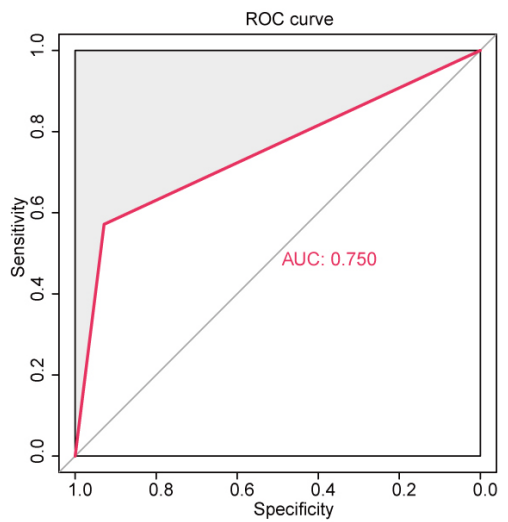

Fig. 5. Comprehensive analysis on the predictive value of the pyroptosis-related risk model. (A) Univariate Cox regression analysis in pyroptosis-related DEGs. (B) LASSO regression analysis. (C) Cross-validation. (D) Multivariate analysis on significant genes. (E) Riskscore in AMI and normal samples. (F) External validation.

\subsection{AMI classifier construction and performance evaluation}

In the univariate analysis, 20 pyroptosis-related genes associated with AMI risk were filtered (Fig. 5A). Following cross-validation in a LASSO regression model (Fig. 5B), 9 genes of vital significance were screened, including NLRP9, GSDMD, CASP8, AIM2, GPX4, NOD1, NOD2, $S C A F 11, G S D M E$ ). Based on the 9 genes, a riskscore was established (Fig. 5C). Further multivariate analysis presented that, CASP8, AIM2, GPX4, NOD1, SCAF11, GS$D M E$ exhibited remarkable significance in predicting the risk of AMI (Fig. 5D, all $p<0.05$ ). AMI patients had significant higher risk scores versus the healthy controls $(p<0.01)$ (Fig. 5E). Based on the riskscore, AMI patients were then classified as high and low risk according to the sextile. The model was further tested in an external dataset (GSE62646) with the ROC-AUC detected to be 0.75 (Fig. 5F), suggesting good performance of the model in predicting AMI risk. Fig. 6 shows the mechanism between AMI and pyroptosis-related genes.

\section{Discussion}

AMI is an irreversible damage to myocardium driven by coronary artery thrombosis or occlusion [23]. Recent studies demonstrated that pyroptosis was involved in the pathogenesis of cardiac injury in AMI [24,25]. Moreover, Inhibition of pyroptosis can cut down myocardial infarct size [26]. In the study, we found that pyroptosis-related genes were highly correlated with infiltrating immune in AMI. In addition, a 9 pyroptosis-related gene based on predictive model for AMI risk was constructed, and identified to have favorable performance in discriminating between high and low risk patients in an external validation set.

A growing number of studies have demonstrated the crucial role of infiltrating immune cells in patients with cardiovascular disease, such as neutrophils, monocyte macrophages and helper T1 cells [27-30]. It remains to be clarified on the abundance of the immune cells in AMI patients. Here, CIBERSORT algorithm was applied to find higher proportions of Plasma cells, T cells CD8 and T cells follicular helper in AMI group versus the healthy control group, which is consistent with the existing studies [31,32]. On $\mathrm{T}$ cells follicular helper, currently there has no relevant report yet and its clinical application value requires 


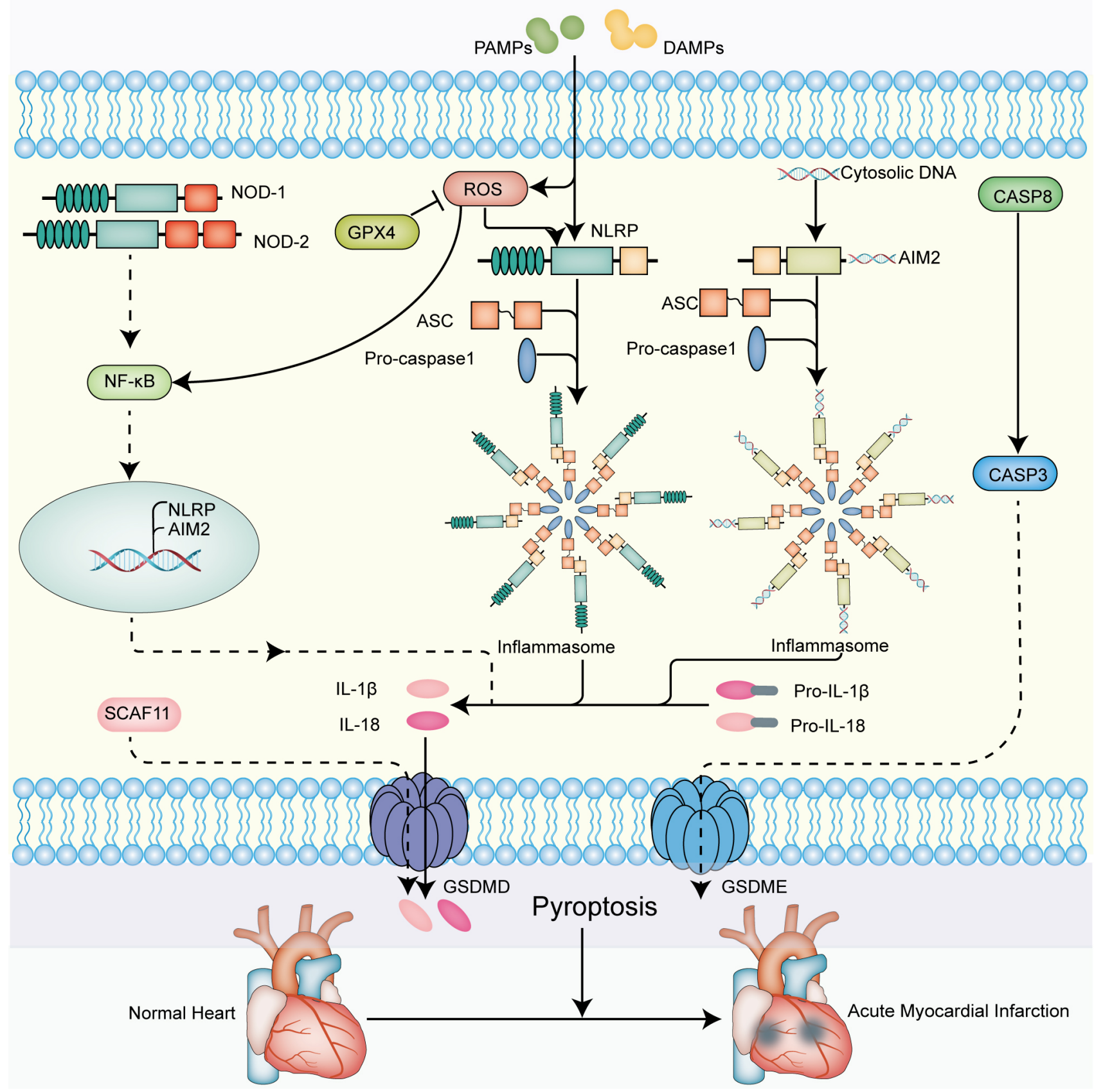

Fig. 6. The molecular signaling mechanisms of pyroptosis-related genes that mediate pathogenesis of AMI. On the one hand, the activation of the intracellular NLRP family is provoked by the various pathogen-associated molecular patterns (PAMPs) and damageassociated molecular patterns (DAMPs), leading to the formation of the inflammasome. On the other hand, the activation of NLRP inflammasome is regulated by ROS, whereas GPX4 suppresses the generation of ROS. Ultimately, the results in releasing of mature $I L-1 \beta, I L-18$ and pyroptotic leakage of intracellular contents. $N O D-1$ and $N O D-2$ activate $N F-\kappa B$, in turn promoting the transcription and translation of AIM2 and NLRP. CASP8 and CASP3 also mediate the pathway of pyroptosis. SCAF11 is reported to contribute to pyroptosis.

further investigation. It was reported that there is certain relationship between cell pyroptosis and immune system function. By releasing TNF- $\alpha$ and IL- $1 \beta$, the incidence of pyroptosis can promote the aggregation of granulocytes, macrophages and dendritic cells [33,34]. We here noted that the pyroptosis-related genes in AMI were remarkably asso- ciated with the infiltrating immune cells in the immune microenvironment. Of note, there was a significantly positive correlation between GZMB and NK cells activated, while an evidently negative association between NLRP 3 and NK cells resting. This demonstrated the close relationship between pyroptosis and NK cells, which was in agreement 
with the literature $[35,36]$. It has been shown in the studies that NK cells are involved in the development and progression of myocardial infarction [37,38], therefore, it may be a potential target for treating AMI. However, further studies will be needed to focus on whether the NK cells might affect AMI via pyroptosis.

The predictive significance for AMI was composed of 9 pyroptosis-related genes, including NLRP9, GSDMD, CASP8, AIM2, GPX4, NOD1, NOD2, SCAF11, GSDME. The use of an external validation set further proved the good performance of the 9-gene signature in predicting the risk of AMI. NLRP9 (NLR Family Pyrin Domain Containing 9) is a member of the NLRs family that comprises a leucine-rich repeat (LRR), nucleotide-bindingoligomerization domain (NACHT), and a caspase activation andrecruitment domain (CARD) or a pyrindomain (PYD). As reported, NLRP9 under inflammation would exhibit stronger expression [39], while knock out of NLRP9 gene could suppress inflammatory reactions and decrease tissue injury [40]. NOD (Nucleotide Binding Oligomerization Domain), including NOD1 and NOD2, shares similar structures to NLRP9 and exerts vital effects during the occurrence of atherosclerosis and further progression to AMI. In an animal experiment, mice of $N O D 1 / 2$ knock-out presented with less lipid deposition and macrophage aggregation as compared to the normal mice [41]. AIM2 (Absent In Melanoma 2) contains a Nterminal PYD signal domain and a C-terminal HIN200 domain, and it can recognize double-stranded DNA. Research revealed that activation of AIM2 inflammasome could aggravate atherosclerosis [42]. The NLRP inflammasome is capable of bringing on the activation of caspase in pyroptosis. Caspase- 8 is a cysteinate-aspartate specific protease and one of the major initiators involved in cell pyroptosis. It would show increasing expression with the occurrence of AMI [43], which is in agreement with the finding of the present study. In cases of myocardial ischemia reperfusion (I/R), Caspase- 8 could increase myocardial NO oxidation and mitochondrial reactive oxygen species (ROS), while the ROS could further mediate pyroptosis via the $N F$ $\kappa B-G S D M D$ signaling pathway $[44,45]$. GSDMD and $G S$ $D M E$ are substrates of Caspase-8, respectively cleaved by Caspase- 1 and lysed by Caspase- 3 upon pyroptosis [46,47]. A latest study presented that, the increasing GSDMD in peripheral blood of AMI patients were cleaved by inflammatory Caspases to release mature $I L-18$ and $I L-1 \beta$, thereby activating cell pyroptosis [48]. Collectively, genes are important players in AMI. GPX4 affects cell metabolism predominantly through regulating mitochondria. Moreover, GPX4 inhibits oxidative stress (such as ROS), which is a crucial signaling pathway involving in pyroptosis [49,50]. $G P X 4$, protecting myocardial cells from injury, was downregulated in AMI [51]. SCAF11 participants in pre-mRNA alternative splicing by modulating the assembly of spliceosomes. Recent studies have identified the involvement of $S C A F 11$ in lipid metabolism and the increased expression in coronary heart disease (2.5 times as likely the control) [52,53]. However, the expression pattern and function of SCAF11 in AMI need to be identified.

There have been multiple biomarkers available for diagnosis and prognosis of AMI. Wang et al. [54] performed weighted gene co-expression network analysis to study potential AMI-related biomarkers. Zhang et al. [55] identified 4 key genes which were capable of diagnosing the plaque changes in recurrent AMI. Nevertheless, there is still a gap on biomarkers predictive for the risk of AMI. Distinct features of AMI were a rapid onset and high mortality. Early revascularization can greatly reduce myocardial injury and the mortality for patients. Thus, early identification and prevention for AMI are extremely important to reduce the incidence and mortality, while improving patient prognosis. In this study, we first conducted a prognostic classifier depending on pyroptosis-related genes in AMI. A previous study found that a novel pyroptosisrelated gene signature had been remarkably effective to predict the prognosis of uveal melanoma [56]. Similarly, pyroptosis-related risk model had been used in order to predict the prognosis of Hepatocellular carcinoma [57]. In the present study, pyroptosis-related gene signature showed promising value in predicting the risk for AMI, moreover, which has a better value as a predictor of AMI through ROC analysis than others [58-62]. The model formula established in this study is simple and easy to implement. Intelligent and accurate early warning of AMI is achieved mainly by collecting blood specimens from people and measuring the gene expression profiles, which will provide a reliable reference basis for clinical decision-making and bring excellent convenience.

This study also has some limitations and shortcomings. First, this study is devised retrospective based on public databases and only one external set was used for validation. In the future, larger clinical sample data are in demand to support the finding of the study. Second, in despite of the good predictive performance of the pyroptosis-related genes we found here, there is paucity of studies on pyroptosis, and the specific biological effects in AMI are largely unknown. Third, the model we established lacks further validation, and in vivo and in vitro experiments as well as clinical trials are required.

\section{Conclusions}

In all, this study uncovered the close relationship between pyroptosis and AMI, and further identified the association between pyroptosis and immune response. The predicting model was built based on pyroptosis-related differential genes. It may provide a new way for predicting the risk of AMI, which has potential clinical value.

\section{Abbreviations}

AMI, acute myocardial infarction; DEGs, differential expressed genes; GEO, Gene Expression Om- 
nibus; LRR, leucine-rich repeat; NACHT, nucleotidebindingoligomerization domain; CARD, caspase activation andrecruitment domain; PYD, pyrindomain; ROS, reactive oxygen species.

\section{Author contributions}

QXM, HC and WHS designed the research study. HHW, QY, JWL and RJ collected and assembled of data. KHG and ZWZ analyzed the data. KHG, ZWZ and PFC wrote the manuscript. All authors contributed to editorial changes in the manuscript. All authors read and approved the final manuscript.

\section{Ethics approval and consent to participate}

Publicly available datasets were downloaded and analyzed in this study. The patients involved in the database have obtained ethical approval. Informed consent and ethical approval were not required for this study in accordance with the national legislation and the institutional requirements.

\section{Acknowledgment}

Not applicable.

\section{Funding}

This study was supported by grants from the National Natural Science Foundation of China (82171698, 82170561, 81741067, 81300279), the Natural Science Foundation for Distinguished Young Scholars of Guangdong Province (2021B1515020003), the Climbing Program of Introduced Talents and High-level Hospital Construction Project of Guangdong Provincial People's Hospital (DFJH201803, KJ012019099, KJ012021143, KY012021183).

\section{Conflict of interest}

The authors declare no conflict of interest.

\section{References}

[1] Ibanez B, James S, Agewall S, Antunes MJ, Bucciarelli-Ducci C, Bueno H, et al. 2017 ESC Guidelines for the management of acute myocardial infarction in patients presenting with STsegment elevation: the Task Force for the management of acute myocardial infarction in patients presenting with ST-segment elevation of the European Society of Cardiology (ESC). European Heart Journal. 2018; 39: 119-177.

[2] Widimsky P, Wijns W, Fajadet J, de Belder M, Knot J, Aaberge $\mathrm{L}$, et al. Reperfusion therapy for ST elevation acute myocardial infarction in Europe: description of the current situation in 30 countries. European Heart Journal. 2010; 31: 943-957.

[3] Collet JP, Thiele H, Barbato E, Barthelemy O, Bauersachs J, Bhatt DL, et al. 2020 ESC Guidelines for the management of acute coronary syndromes in patients presenting without persistent ST-segment elevation. European Heart Journal. 2021; 42: 1289-1367.

[4] Alfonso F, Gonzalo N, Rivero F, Escaned J. The year in cardiovascular medicine 2020: interventional cardiology. European Heart Journal. 2021; 42: 985-1003.
[5] Chandrashekhar Y, Alexander T, Mullasari A, Kumbhani DJ, Alam S, Alexanderson E, et al. Resource and InfrastructureAppropriate Management of ST-Segment Elevation Myocardial Infarction in Low- and Middle-Income Countries. Circulation. 2020; 141: 2004-2025.

[6] Cooper JG, Ferguson J, Donaldson LA, Black KMM, Horrill JL, Davidson EM, et al. Could High-Sensitivity Cardiac Troponin Testing Rule Out Acute Myocardial Infarction in the Prehospital Setting? Journal of the American College of Cardiology. 2021; 78: 2392-2394.

[7] de Winter RJ, Koster RW, Sturk A, Sanders GT. Value of myoglobin, troponin $\mathrm{T}$, and CK-MBmass in ruling out an acute myocardial infarction in the emergency room. Circulation. 1995; 92: 3401-3407.

[8] Thygesen K, Alpert JS, Jaffe AS, Chaitman BR, Bax JJ, Morrow DA, et al. Fourth Universal Definition of Myocardial Infarction (2018). Global Heart. 2018; 13: 305-338.

[9] Ge Y, Wang TJ. Identifying novel biomarkers for cardiovascular disease risk prediction. Journal of Internal Medicine. 2012; 272: 430-439.

[10] Wang Q, Wu J, Zeng Y, Chen K, Wang C, Yang S, et al. Pyroptosis: a pro-inflammatory type of cell death in cardiovascular disease. Clinica Chimica Acta. 2020; 510: 62-72.

[11] Bedoui S, Herold MJ, Strasser A. Emerging connectivity of programmed cell death pathways and its physiological implications. Nature Reviews Molecular Cell Biology. 2020; 21: 678-695.

[12] Sandanger Ø, Ranheim T, Vinge LE, Bliksøen M, Alfsnes K, Finsen AV, et al. The NLRP3 inflammasome is up-regulated in cardiac fibroblasts and mediates myocardial ischaemiareperfusion injury. Cardiovascular Research. 2013; 99: 164 174.

[13] Mezzaroma E, Toldo S, Farkas D, Seropian IM, Van Tassell BW, Salloum FN, et al. The inflammasome promotes adverse cardiac remodeling following acute myocardial infarction in the mouse. Proceedings of the National Academy of Sciences of the United States of America. 2011; 108: 19725-19730.

[14] Han Y, Dong B, Chen M, Yao C. LncRNA H19 suppresses pyroptosis of cardiomyocytes to attenuate myocardial infarction in a PBX3CYP1B1-dependent manner. Molecular and Cellular Biochemistry. 2021; 476: 1387-1400.

[15] Liu Y, Li P, Qiao C, Wu T, Sun X, Wen M, et al. Chitosan Hydrogel Enhances the Therapeutic Efficacy of Bone MarrowDerived Mesenchymal Stem Cells for Myocardial Infarction by Alleviating Vascular Endothelial Cell Pyroptosis. Journal of Cardiovascular Pharmacology. 2020; 75: 75-83.

[16] Zhou C, Zhan G, Jin Y, Chen J, Shen Z, Shen Y, et al. A novel pyroptosis-related gene signature to predict outcomes in laryngeal squamous cell carcinoma. Aging. 2021; 13: 25960-25979.

[17] Zhang Y, He R, Lei X, Mao L, Jiang P, Ni C, et al. A Novel Pyroptosis-Related Signature for Predicting Prognosis and Indicating Immune Microenvironment Features in Osteosarcoma. Frontiers in Genetics. 2021; 12: 780780.

[18] Ye Y, Dai Q, Qi H. A novel defined pyroptosis-related gene signature for predicting the prognosis of ovarian cancer. Cell Death Discovery. 2021; 7: 71.

[19] Ritchie ME, Phipson B, Wu D, Hu Y, Law CW, Shi W, et al. Limma powers differential expression analyses for RNAsequencing and microarray studies. Nucleic Acids Research. 2015; 43: e47.

[20] Newman AM, Liu CL, Green MR, Gentles AJ, Feng W, Xu Y, et al. Robust enumeration of cell subsets from tissue expression profiles. Nature Methods. 2015; 12: 453-457.

[21] Mallick H, Alhamzawi R, Paul E, Svetnik V. The reciprocal Bayesian LASSO. Statistics in Medicine. 2021; 40: 4830-4849.

[22] Li X, Gao Y, Xu Z, Zhang Z, Zheng Y, Qi F. Identification 
of prognostic genes in adrenocortical carcinoma microenvironment based on bioinformatic methods. Cancer Medicine. 2020; 9: 1161-1172.

[23] Heusch G. Myocardial ischaemia-reperfusion injury and cardioprotection in perspective. Nature Reviews Cardiology. 2020; 17 : 773-789.

[24] Li Z, Xu H, Liu X, Hong Y, Lou H, Liu H, et al. GDF11 inhibits cardiomyocyte pyroptosis and exerts cardioprotection in acute myocardial infarction mice by upregulation of transcription factor HOXA3. Cell Death \& Disease. 2020; 11: 917.

[25] Zhang K, Shen X, Wang M, Wang L, Sun H, Li X, et al. Retinol-Binding Protein 4 Promotes Cardiac Injury after Myocardial Infarction via Inducing Cardiomyocyte Pyroptosis through an Interaction with NLRP3. Journal of the American Heart Association. 2021; 10: e022011.

[26] Liu W, Shen J, Li Y, Wu J, Luo X, Yu Y, et al. Pyroptosis inhibition improves the symptom of acute myocardial infarction. Cell Death \& Disease. 2021; 12: 852.

[27] Silvestre-Roig C, Braster Q, Ortega-Gomez A, Soehnlein O. Neutrophils as regulators of cardiovascular inflammation. Nature Reviews Cardiology. 2020; 17: 327-340.

[28] Newby LK. Inflammation as a Treatment Target after Acute Myocardial Infarction. New England Journal of Medicine. 2019; 381: 2562-2563.

[29] Gisterå A, Hansson GK. The immunology of atherosclerosis. Nature Reviews. Nephrology. 2017; 13: 368-380.

[30] Fernandez DM, Giannarelli C. Immune cell profiling in atherosclerosis: role in research and precision medicine. Nature Reviews Cardiology. 2022; 19: 43-58.

[31] Zhang L, Wang Z, Wang D, Zhu J, Wang Y. CD8+CD28+ T cells might mediate injury of cardiomyocytes in acute myocardial infarction. Molecular Immunology. 2018; 101: 74-79.

[32] Kyaw T, Loveland P, Kanellakis P, Cao A, Kallies A, Huang AL, et al. Alarmin-activated $\mathrm{B}$ cells accelerate murine atherosclerosis after myocardial infarction via plasma cell-immunoglobulindependent mechanisms. European Heart Journal. 2021; 42: 938-947.

[33] Loveless R, Bloomquist R, Teng Y. Pyroptosis at the forefront of anticancer immunity. Journal of Experimental \& Clinical Cancer Research. 2021; 40: 264.

[34] Li L, Jiang M, Qi L, Wu Y, Song D, Gan J, et al. Pyroptosis, a new bridge to tumor immunity. Cancer Science. 2021; 112: 3979-3994.

[35] Maltez VI, Tubbs AL, Cook KD, Aachoui Y, Falcone EL, Holland SM, et al. Inflammasomes Coordinate Pyroptosis and Natural Killer Cell Cytotoxicity to Clear Infection by a Ubiquitous Environmental Bacterium. Immunity. 2015; 43: 987-997.

[36] Hage C, Hoves S, Strauss L, Bissinger S, Prinz Y, Pöschinger T, et al. Sorafenib Induces Pyroptosis in Macrophages and Triggers Natural Killer Cell-Mediated Cytotoxicity against Hepatocellular Carcinoma. Hepatology. 2019; 70: 1280-1297.

[37] Kologrivova I, Shtatolkina M, Suslova T, Ryabov V. Cells of the Immune System in Cardiac Remodeling: Main Players in Resolution of Inflammation and Repair After Myocardial Infarction. Frontiers in Immunology. 2021; 12: 664457.

[38] Knorr M, Münzel T, Wenzel P. Interplay of NK cells and monocytes in vascular inflammation and myocardial infarction. Frontiers in Physiology. 2014; 5: 295.

[39] Nagyőszi P, Nyúl-Tóth Á, Fazakas C, Wilhelm I, Kozma M, Molnár J, et al. Regulation of NOD-like receptors and inflammasome activation in cerebral endothelial cells. Journal of Neurochemistry. 2015; 135: 551-564.

[40] Yanling Q, Xiaoning C, Fei B, Liyun F, Huizhong H, Daqing S. Inhibition of NLRP9b attenuates acute lung injury through suppressing inflammation, apoptosis and oxidative stress in murine and cell models. Biochemical and Biophysical Research Com- munications. 2018; 503: 436-443.

[41] Vlacil A, Schuett J, Ruppert V, Soufi M, Oberoi R, Shahin K, et al. Deficiency of Nucleotide-binding oligomerization domaincontaining proteins (NOD) 1 and 2 reduces atherosclerosis. Basic Research in Cardiology. 2020; 115: 47.

[42] Fidler TP, Xue C, Yalcinkaya M, Hardaway B, Abramowicz S, Xiao T, et al. The AIM2 inflammasome exacerbates atherosclerosis in clonal haematopoiesis. Nature. 2021; 592: 296-301.

[43] Milbank E, Soleti R, Martinez E, Lahouel B, Hilairet G, Martinez MC, et al. Microparticles from apoptotic RAW 264.7 macrophage cells carry tumour necrosis factor-alpha functionally active on cardiomyocytes from adult mice. Journal of Extracellular Vesicles. 2015; 4: 28621.

[44] Fauconnier J, Meli AC, Thireau J, Roberge S, Shan J, Sassi Y, et al. Ryanodine receptor leak mediated by caspase- 8 activation leads to left ventricular injury after myocardial ischemiareperfusion. Proceedings of the National Academy of Sciences of the United States of America. 2011; 108: 13258-13263.

[45] Lei Q, Yi T, Chen C. NF-kappaB-Gasdermin D (GSDMD) Axis Couples Oxidative Stress and NACHT, LRR and PYD Domains-Containing Protein 3 (NLRP3) InflammasomeMediated Cardiomyocyte Pyroptosis Following Myocardial Infarction. Medical Science Monitor. 2018; 24: 6044-6052.

[46] Kesavardhana S, Malireddi RKS, Kanneganti T. Caspases in Cell Death, Inflammation, and Pyroptosis. Annual Review of Immunology. 2020; 38: 567-595.

[47] Liu X, Xia S, Zhang Z, Wu H, Lieberman J. Channelling inflammation: gasdermins in physiology and disease. Nature Reviews Drug Discovery. 2021; 20: 384-405.

[48] Shi H, Gao Y, Dong Z, Yang J, Gao R, Li X, et al. GSDMDMediated Cardiomyocyte Pyroptosis Promotes Myocardial I/R Injury. Circulation Research. 2021; 129: 383-396.

[49] Jaén RI, Val-Blasco A, Prieto P, Gil-Fernández M, Smani T, López-Sendón JL, et al. Innate Immune Receptors, Key Actors in Cardiovascular Diseases. JACC: Basic to Translational Science. 2020; 5: 735-749.

[50] Li P, Chang M. Roles of PRR-Mediated Signaling Pathways in the Regulation of Oxidative Stress and Inflammatory Diseases. International Journal of Molecular Sciences. 2021; 22: 7688.

[51] Park T, Park JH, Lee GS, Lee J, Shin JH, Kim MW, et al. Quantitative proteomic analyses reveal that GPX4 downregulation during myocardial infarction contributes to ferroptosis in cardiomyocytes. Cell Death \& Disease. 2019; 10: 835.

[52] Damián-Zamacona S, Toledo-Ibelles $\mathrm{P}$, Ibarra-Abundis $\mathrm{MZ}$, Uribe-Figueroa L, Hernández-Lemus E, Macedo-Alcibia KP, et al. Early Transcriptomic Response to LDL and oxLDL in Human Vascular Smooth Muscle Cells. PloS one. 2016; 11: e0163924.

[53] Pan Z, Fan Z, Ma J, Liu H, Shen L, He B, et al. Profiling and functional characterization of circulation LncRNAs that are associated with coronary atherosclerotic plaque stability. American Journal of Translational Research. 2019; 11: 3801-3815.

[54] Wang Y, Zhang X, Duan M, Zhang C, Wang K, Feng L, et al. Identification of Potential Biomarkers Associated with Acute Myocardial Infarction by Weighted Gene Coexpression Network Analysis. Oxidative Medicine and Cellular Longevity. 2021; 2021: 1-11.

[55] Zhang Q, Zheng Y, Ning M, Li T. KLRD1, FOSL2 and LILRB3 as potential biomarkers for plaques progression in acute myocardial infarction and stable coronary artery disease. BMC Cardiovascular Disorders. 2021; 21: 344.

[56] Cao Y, Xie J, Chen L, Hu Y, Zhai L, Yuan J, et al. Construction and Validation of a Novel Pyroptosis-Related Gene Signature to Predict the Prognosis of Uveal Melanoma. Frontiers in Cell and Developmental Biology. 2021; 9: 761350.

[57] Wu Q, Jiang S, Cheng T, Xu M, Lu B. A Novel Pyroptosis- 
related Prognostic Model for Hepatocellular Carcinoma. Frontiers in Cell and Developmental Biology 2021; 9: 770301.

[58] Koulouri A, Darioli R, Dine Qanadli S, Katz E, Eeckhout E, Mazzolai L, et al. The atherosclerosis burden score. Vasa. 2021; 50: $280-285$.

[59] Li M, Wang Y, Yang X, Xu L, Li W, Xia K, et al. Circulating Long Noncoding RNA LIPCAR Acts as a Novel Biomarker in Patients with ST-Segment Elevation Myocardial Infarction. Medical Science Monitor. 2018; 24: 5064-5070.

[60] Wu Q, Chen Y, Chen S, Wu X, Nong W. Correlation between adiponectin, chemerin, vascular endothelial growth factor and epicardial fat volume in patients with coronary artery disease. Experimental and Therapeutic Medicine. 2020; 19: 1095-1102.

[61] Yan Z, Wang X, Liu YS, Xing XW, Zhang XG, Lu QH. Decreased serum SIRT6 as a novel predictor of coronary artery disease. European Review for Medical and Pharmacological Sciences. 2021; 25: 6660-6669.

[62] Zheng Y, Lang Y, Qi Z, Gao W, Hu X, Li T. PIK3R1, SPNB2, and CRYAB as Potential Biomarkers for Patients with Diabetes and Developing Acute Myocardial Infarction. International Journal of Endocrinology. 2021; 2021: 2267736. 